Int. J. Contemp. Math. Sciences, Vol. 2, 2007, no. 21, 999 - 1008

\title{
Term Extensions of Partial Ring-Like Quantum Logics
}

\author{
Dietmar Dorninger and Helmut Länger
}

\author{
Vienna University of Technology \\ Institute of Discrete Mathematics and Geometry \\ Wiedner Hauptstraße 8-10, 1040 Vienna, Austria \\ dietmar.dorninger@tuwien.ac.at h.laenger@tuwien.ac.at
}

\begin{abstract}
The one-to-one correspondence between Boolean algebras and Boolean rings can be generalized to a mapping from partial ring-like algebras to bounded lattices with an antitone involution (*-lattices), cf. [1], [2]. These partial algebras are known as partial generalized Boolean quasirings (pGBQRs) and can be used to construct various kinds of quantum logics (cf. [1] - [6]). To a large extent this is due to the fact that the partial operation $\oplus$ of pGBQRs can be extended almost arbitrarily to a full operation + . However, in view of Boolean rings and Boolean algebras it is evident to demand that + should be a term built up by the operations of the corresponding *-lattice. How this can be achieved is investigated as well for the case that the underlying lattice is an arbitrary *-lattice as for the special case of de Morgan algebras. Moreover, we study various classes of pGBQRs such that + is associative.
\end{abstract}

Mathematics Subject Classification: 08A40, 06C15, 06D30

Keywords: Generalized Boolean quasiring, *-lattice, term, normal form, de Morgan algebra, associativity

\section{Introduction}

In order to generalize the one-to-one correspondence between Boolean algebras and Boolean rings to arbitrary bounded lattices with an antitone involution * (in the following called *-lattices) generalized Boolean quasirings (GBQRs) and partial algebras, which can be extended to GBQRs, so-called partial generalized Boolean quasirings (pGBQRs), have been introduced (cf. [1] and [2]). pGBQRs and *-lattices are in one-to-one correspondence and can be somehow identified. Many classes of GBQRs turned out to provide 
for a large variety of presumptive quantum-logics (including logics which can be defined by means of Mackey's probability function), cf. [1] - [6]. A crucial point in constructing appropriate GBQRs from pGBQRs is the extension of the (only) partial operation $\oplus$ of pGBQRs to a full operation + of the GBQRs that arise. There the representation of + by means of *-lattice terms $t$ plays an important role, as we know from the correspondence between Boolean algebras and Boolean rings where $x+y$ is defined by the *-lattice term $t(x, y)=\left(x \wedge y^{*}\right) \vee\left(x^{*} \wedge y\right)=(x \vee y) \wedge\left(x^{*} \vee y^{*}\right)$. From what we have already said it will also be plausible (and explained in detail later on) to assume that the considered terms $t$ have the following properties:

$(\alpha) \quad t(x, y)=t(y, x)$, i. e. + should be commutative.

( $\beta) \quad t(x, 0)=x$ and $t(x, 1)=x^{*}$, i. e. $x+0=x$ and $x+1=x^{*}$ for all $x$.

In particular, $(\beta)$ implies the weaker version

$\left(\beta_{0}\right) \quad t(0,0)=t(1,1)=0$ and $t(0,1)=t(1,0)=1$.

Both of the following representations of the symmetric difference in Boolean algebras,

$$
\begin{aligned}
& x+{ }_{1} y:=\left(x \wedge y^{*}\right) \vee\left(x^{*} \wedge y\right) \text { and } \\
& x+{ }_{2} y:=(x \vee y) \wedge\left(x^{*} \vee y^{*}\right), \text { respectively, }
\end{aligned}
$$

fulfil $(\alpha)$ and $(\beta)$. (In general, $x+{ }_{1} y \neq x+{ }_{2} y$ in *-lattices.)

First, we will prove that for arbitrary *-lattices assumption $\left(\beta_{0}\right)$ is equivalent to $x+{ }_{1} y \leq t(x, y) \leq x+{ }_{2} y$ from which we can conclude by a theorem of M. Navara ([9]) that in case a pGBQR corresponds to an orthomodular lattice the only possible extensions to GBQRs by means of *-lattice terms that satisfy $(\alpha)$ and $(\beta)$ are $+_{1}$ and $+_{2}$. Next we show that for de Morgan algebras the situation is the same, however we will derive this result by different means. We then turn our attention to associativity, i. e. we assume (as in the case of Boolean rings)

$$
t(t(x, y), z)=t(x, t(y, z)) \text { for all } x, y, z .
$$

We introduce a (large) class of *-lattices (in fact a variety) that only admit associative term extensions of $\oplus$.

\section{Basic definitions, upper and lower bounds}

Definition 2.1 (cf. [2]). A partial algebra $(R, \oplus, \cdot, 0,1)$ of type $(2,2,0,0)$ with a partial operation $\oplus:\{0,1\} \times R \rightarrow R$ and a total operation $\cdot$ is called a partial 
generalized Boolean quasiring (pGBQR), if the following laws hold:

$$
\begin{aligned}
& 0 \oplus x=x \\
& (x y) z=x(y z) \\
& x y=y x \\
& x x=x \\
& x 0=0 \\
& x 1=x \\
& 1 \oplus(1 \oplus x y)(1 \oplus x)=x
\end{aligned}
$$

For every fixed set $R$ the formulas

$$
\begin{aligned}
& x \vee y:=1 \oplus(1 \oplus x)(1 \oplus y), \\
& x \wedge y:=x y, \\
& x^{*}:=1 \oplus x,
\end{aligned}
$$

respectively

$$
\begin{aligned}
& 0 \oplus x:=x, \\
& 1 \oplus x:=x^{*}, \\
& x y:=x \wedge y
\end{aligned}
$$

induce mutually inverse bijections between the set of all pGBQRs on $R$ and the set of all *-lattices on $R$ (cf. [2]), which, from now on, will be identified.

If the operation $\oplus$ of a $\operatorname{pGBQR}(R, \oplus, \cdot, 0,1)$ is extended to a full operation + such that

$$
x+y=y+x
$$

for all $x, y \in R$, then the pGBQR becomes a GBQR. Extensions + of this kind always exist (in affluence), because putting for all $x \in R 0+x=x+0:=0 \oplus x$ and $1+x=x+1:=1 \oplus x$ and for all $x, y \in R \backslash\{0,1\} x+y=y+x$ arbitrarily, we obtain a GBQR.

Our goal is to consider such extensions + for which $x+y=t(x, y)$ is a *-lattice term. Hence we assume $(\alpha)$ and $(\beta)$.

The operations $+_{1}$ and $+_{2}$ are canonical examples of such extensions.

In the following $\mathbf{L}^{*}$ should always denote the variety of ${ }^{*}$-lattices and $t$ a binary *-lattice term by means of which a *-lattice, considered as a pGBQR, can be extended to a GBQR. This means that $t$ has to fulfil $(\alpha)$ and $(\beta)$, which we always assume from now on if not explicitly stated otherwise.

Extensions of $\oplus$ that satisfy $(\alpha)$ and $(\beta)$ can also be achieved by terms in more than two variables with the additional variables considered as parameters which can be substituted by certain kinds of elements of a *-lattice. This means we then deal with a parameterized term extension or polynomial (in the sense of [8]) extension, respectively. For example, given an extension $t(x, y)$ that fulfils $(\alpha)$ and $(\beta) t_{z}(x, y):=(t(x, y) \wedge z) \vee\left(t(x, y) \wedge z^{*}\right)$ is an extension of $\oplus$ for any fixed central element $z$ of a ${ }^{*}$-lattice, and $t_{z}(x, y)=t_{z}(y, x), t_{z}(x, 0)=x$, 
$t_{z}(x, 1)=x^{*}$. Though we will not further persue these kinds of extensions the next theorem also comprises this more general situation.

First we agree on the following notation: For a ${ }^{*}$-lattice $\mathcal{L}=\left(L, \vee, \wedge,{ }^{*}, 0,1\right)$ and for $a \in L$ we put $a^{1}:=a$ and $a^{-1}:=a^{*}$. Then for all $a \in L$ and $i, j \in\{-1,1\}\left(a^{i}\right)^{j}=a^{i j}$ and hence

$$
\left(0^{i}\right)^{j}=0 \Leftrightarrow\left(1^{i}\right)^{j}=1 \Leftrightarrow i=j
$$

Theorem 2.2 For $f:\{0,1\}^{n} \rightarrow\{0,1\}$ an n-ary term $t$ in $\mathbf{L}^{*}$ satisfies $t \mid\{0,1\}^{n}=$ $f$ if and only if

$$
\begin{array}{r}
t \in\left[\bigvee_{i_{1}, \ldots, i_{n} \in\{-1,1\}}\left(f\left(1^{i_{1}}, \ldots, 1^{i_{n}}\right) \wedge x_{1}^{i_{1}} \wedge \ldots \wedge x_{n}^{i_{n}}\right),\right. \\
\left.\bigwedge_{i_{1}, \ldots, i_{n} \in\{-1,1\}}\left(f\left(0^{i_{1}}, \ldots, 0^{i_{n}}\right) \vee x_{1}^{i_{1}} \vee \ldots \vee x_{n}^{i_{n}}\right)\right] .
\end{array}
$$

Remark 2.3 The expressions occurring at the end of Theorem 2.2 are exactly the disjunctive respectively conjunctive normal form in case of Boolean algebras.

Proof of Theorem 2.2 First assume $t \mid\{0,1\}^{n}=f$. According to the de Morgan laws there exists a $2 n$-ary term function $s$ on $(L, \vee, \wedge, 0,1)$ such that

$$
s\left(x_{1}, \ldots, x_{n}, x_{1}^{*}, \ldots, x_{n}^{*}\right)=t\left(x_{1}, \ldots, x_{n}\right) .
$$

Let $j_{1}, \ldots, j_{n} \in\{-1,1\}$ and assume $f\left(1^{j_{1}}, \ldots, 1^{j_{n}}\right)=1$. For $i=1, \ldots, n$ put

$$
\left(y_{i}, y_{n+i}\right):=\left\{\begin{array}{l}
\left(x_{i}, 0\right)=\left(x_{i}^{j_{i}}, 0\right) \\
\left(0, x_{i}^{*}\right)=\left(0, x_{i}^{j_{i}}\right)
\end{array}\right\} \text { if } j_{i}=\left\{\begin{array}{r}
1 \\
-1
\end{array}\right.
$$

Then

$$
t\left(x_{1}, \ldots, x_{n}\right)=s\left(x_{1}, \ldots, x_{n}, x_{1}^{*}, \ldots, x_{n}^{*}\right) \geq s\left(y_{1}, \ldots, y_{2 n}\right)
$$

and $s\left(y_{1}, \ldots, y_{2 n}\right) \geq x_{1}^{j_{1}} \wedge \ldots \wedge x_{n}^{j_{n}}$ or $s\left(y_{1}, \ldots, y_{2 n}\right)=0$. The latter would imply

$$
1=f\left(1^{j_{1}}, \ldots, 1^{j_{n}}\right)=t\left(1^{j_{1}}, \ldots, 1^{j_{n}}\right)=s\left(1^{j_{1}}, \ldots, 1^{j_{n}}, 0^{j_{1}}, \ldots, 0^{j_{n}}\right)=0,
$$

a contradiction. Hence $s\left(y_{1}, \ldots, y_{2 n}\right) \geq x_{1}^{j_{1}} \wedge \ldots \wedge x_{n}^{j_{n}}$ and therefore

$t\left(x_{1}, \ldots, x_{n}\right) \geq s\left(y_{1}, \ldots, y_{2 n}\right) \geq x_{1}^{j_{1}} \wedge \ldots \wedge x_{n}^{j_{n}}=f\left(1^{j_{1}}, \ldots, 1^{j_{n}}\right) \wedge x_{1}^{j_{1}} \wedge \ldots \wedge x_{n}^{j_{n}}$

If $f\left(1^{j_{1}}, \ldots, 1^{j_{n}}\right)=0$ then obviously

$$
t\left(x_{1}, \ldots, x_{n}\right) \geq f\left(1^{j_{1}}, \ldots, 1^{j_{n}}\right) \wedge x_{1}^{j_{1}} \wedge \ldots \wedge x_{n}^{j_{n}} .
$$


Hence in any case

$$
t\left(x_{1}, \ldots, x_{n}\right) \geq f\left(1^{j_{1}}, \ldots, 1^{j_{n}}\right) \wedge x_{1}^{j_{1}} \wedge \ldots \wedge x_{n}^{j_{n}} .
$$

Therefore

$$
t\left(x_{1}, \ldots, x_{n}\right) \geq \bigvee_{i_{1}, \ldots, i_{n} \in\{-1,1\}}\left(f\left(1^{i_{1}}, \ldots, 1^{i_{n}}\right) \wedge x_{1}^{i_{1}} \wedge \ldots \wedge x_{n}^{i_{n}}\right) .
$$

The upper bound is obtained by dual arguments.

If, conversely,

$$
\begin{array}{r}
t \in\left[\bigvee_{i_{1}, \ldots, i_{n} \in\{-1,1\}}\left(f\left(1^{i_{1}}, \ldots, 1^{i_{n}}\right) \wedge x_{1}^{i_{1}} \wedge \ldots \wedge x_{n}^{i_{n}}\right),\right. \\
\left.\bigwedge_{i_{1}, \ldots, i_{n} \in\{-1,1\}}\left(f\left(0^{i_{1}}, \ldots, 0^{i_{n}}\right) \vee x_{1}^{i_{1}} \vee \ldots \vee x_{n}^{i_{n}}\right)\right]
\end{array}
$$

and $j_{1}, \ldots, j_{n} \in\{-1,1\}$ then

$$
\begin{aligned}
f\left(1^{j_{1}}, \ldots, 1^{j_{n}}\right) & =\bigvee_{i_{1}, \ldots, i_{n} \in\{-1,1\}}\left(f\left(1^{i_{1}}, \ldots, 1^{i_{n}}\right) \wedge\left(1^{j_{1}}\right)^{i_{1}} \wedge \ldots \wedge\left(1^{j_{n}}\right)^{i_{n}}\right)= \\
& =\left(\bigvee_{i_{1}, \ldots, i_{n} \in\{-1,1\}}\left(f\left(1^{i_{1}}, \ldots, 1^{i_{n}}\right) \wedge x_{1}^{i_{1}} \wedge \ldots \wedge x_{n}^{i_{n}}\right)\right)\left(1^{j_{1}}, \ldots, 1^{j_{n}}\right) \leq \\
& \leq t\left(1^{j_{1}}, \ldots, 1^{j_{n}}\right) \leq \\
& \leq\left(\bigwedge_{i_{1}, \ldots, i_{n} \in\{-1,1\}}\left(f\left(0^{i_{1}}, \ldots, 0^{i_{n}}\right) \vee x_{1}^{i_{1}} \vee \ldots \vee x_{n}^{i_{n}}\right)\right)\left(1^{j_{1}}, \ldots, 1^{j_{n}}\right)= \\
& =\bigwedge_{i_{1}, \ldots, i_{n} \in\{-1,1\}}\left(f\left(0^{i_{1}}, \ldots, 0^{i_{n}}\right) \vee\left(1^{j_{1}}\right)^{i_{1}} \vee \ldots \vee\left(1^{j_{n}}\right)^{i_{n}}\right)= \\
& =f\left(0^{-j_{1}}, \ldots, 0^{-j_{n}}\right)=f\left(1^{j_{1}}, \ldots, 1^{j_{n}}\right),
\end{aligned}
$$

i. e. $t \mid\{0,1\}^{n}=f$.

If $x+{ }_{1} y \leq t(x, y) \leq x+{ }_{2} y$ for all $x, y \in L$, we will write $+\in\left[+_{1},+_{2}\right]$ or, equivalently, if $x+y=t(x, y),+_{1} \leq+\leq+_{2}$.

Corollary 2.4 A binary term $t$ in $\mathbf{L}^{*}$ satisfies $\left(\beta_{0}\right)$ if and only if $t \in\left[+_{1},+_{2}\right]$.

Corollary 2.5 Within $\mathbf{L}^{*}$ for all term extensions + of pGBQRs to GBQRs $+_{1} \leq+\leq+_{2}$.

Corollary 2.6 Within the variety of orthomodular lattices all term extensions of pGBQRs to GBQRs are given by $+_{1}$ and $+_{2}$.

Proof Because of $+_{1}=+_{2}$ in Boolean algebras, by Corollary 2.5 the term has to coincide with the symmetric difference in Boolean algebras. As shown by M. Navara ([9]), in orthomodular lattices there are six binary terms that coincide with the symmetric difference in case of Boolean algebras, four noncommutative and $+_{1}$ and $+_{2}$.

A trivial consequence of Corollary 2.6 is 
Corollary 2.7 Within the variety of Boolean algebras there exists only one possible term extension of pGBQRs to GBQRs, namely $+_{1}=+_{2}$, which leads to a Boolean ring, and hence is associative.

\section{De Morgan algebras associated to pGBQRs}

We recall that a de Morgan algebra is a distributive *-lattice.

Theorem 3.1 In the variety of de Morgan algebras there are exactly four binary terms satisfying $\left(\beta_{0}\right)$, namely

$$
x+{ }_{1} y,\left(x+{ }_{1} y\right) \vee\left(x \wedge x^{*}\right),\left(x+{ }_{1} y\right) \vee\left(y \wedge y^{*}\right) \text { and } x+{ }_{2} y .
$$

Proof By D. Dorninger and D. Schweigert (cf. [7]) the binary terms in the variety of de Morgan algebras are of the form

$$
\begin{aligned}
t(x, y)= & \left(a_{1111} \wedge x \wedge x^{*} \wedge y \wedge y^{*}\right) \vee\left(a_{1110} \wedge x \wedge x^{*} \wedge y\right) \vee \\
& \vee\left(a_{1101} \wedge x \wedge x^{*} \wedge y^{*}\right) \vee\left(a_{1100} \wedge x \wedge x^{*}\right) \vee\left(a_{1011} \wedge x \wedge y \wedge y^{*}\right) \vee \\
& \vee\left(a_{1010} \wedge x \wedge y\right) \vee\left(a_{1001} \wedge x \wedge y^{*}\right) \vee\left(a_{1000} \wedge x\right) \vee \\
& \vee\left(a_{0111} \wedge x^{*} \wedge y \wedge y^{*}\right) \vee\left(a_{0110} \wedge x^{*} \wedge y\right) \vee\left(a_{0101} \wedge x^{*} \wedge y^{*}\right) \vee \\
& \vee\left(a_{0100} \wedge x^{*}\right) \vee\left(a_{0011} \wedge y \wedge y^{*}\right) \vee\left(a_{0010} \wedge y\right) \vee\left(a_{0001} \wedge y^{*}\right) \vee a_{0000}
\end{aligned}
$$

where $a_{i_{1} i_{2} i_{3} i_{4}} \in\{0,1\}$ for all $i_{1}, i_{2}, i_{3}, i_{4} \in\{0,1\}$ and $a_{i_{1} i_{2} i_{3} i_{4}} \geq a_{j_{1} j_{2} j_{3} j_{4}}$ whenever $\left(i_{1}, i_{2}, i_{3}, i_{4}\right) \geq\left(j_{1}, j_{2}, j_{3}, j_{4}\right)$ in $\left(\{0,1\}^{4}, \leq\right)$. Now assume $\left(\beta_{0}\right) . t(0,0)=$ $t(1,1)=0$ implies

$$
a_{1010}=a_{1000}=a_{0101}=a_{0100}=a_{0010}=a_{0001}=a_{0000}=0
$$

and $t(0,1)=t(1,0)=1$ yields $a_{1001}=a_{0110}=1$ from which we can conclude

$$
a_{1111}=a_{1110}=a_{1101}=a_{1011}=a_{0111}=1 .
$$

Since

$$
\begin{gathered}
\left(x \wedge x^{*} \wedge y \wedge y^{*}\right) \vee\left(x \wedge x^{*} \wedge y\right) \vee\left(x \wedge x^{*} \wedge y^{*}\right) \vee\left(x \wedge y \wedge y^{*}\right) \vee\left(x \wedge y^{*}\right) \vee \\
\vee\left(x^{*} \wedge y \wedge y^{*}\right) \vee\left(x^{*} \wedge y\right)=x+{ }_{1} y \\
\left(x+{ }_{1} y\right) \vee\left(x \wedge x^{*}\right) \vee\left(y \wedge y^{*}\right)=x+{ }_{2} y
\end{gathered}
$$

it follows

$$
t \in\left\{+_{1},\left(x+{ }_{1} y\right) \vee\left(x \wedge x^{*}\right),\left(x+{ }_{1} y\right) \vee\left(y \wedge y^{*}\right),+_{2}\right\}
$$

Corollary 3.2 Within the variety of de Morgan algebras all term extensions of pGBQRs to GBQRs are given by $+_{1}$ and $+_{2}$. 


\section{Associative extensions}

First we ask for classes of ${ }^{*}$-lattices such that every ${ }^{*}$-lattice term extension $x+y=t(x, y)$ is associative.

As shown in [4], $+\in\left[+_{1},+_{2}\right]$ together with the associativity of + implies that the ${ }^{*}$-lattice has to be ${ }^{*}$-modular, i. e. $x \leq y$ implies $\left(x \vee x^{*}\right) \wedge y=x \vee\left(x^{*} \wedge y\right)$ or equivalently (see [4]) $x \leq y \leq z$ and $y \leq x \vee x^{*}$ together imply $\left(x \vee y^{*}\right) \wedge z=$ $x \vee\left(y^{*} \wedge z\right)$. In the special case of the variety of ortholattices one obtains that there is exactly one subclass of this variety such that every *-lattice term extension of its members is associative, namely the class of Boolean algebras (cf. also [4]). So the emphasis will be on ${ }^{*}$-lattices such that $x \wedge x^{*} \neq 0$ in general.

Lemma 4.1 If for all elements $x, y, z$ of $a^{*}$-lattice

$$
\begin{aligned}
& \left(\left((x \vee y) \wedge\left(x^{*} \vee y^{*}\right)\right) \vee z\right) \wedge\left(\left(\left(x \vee y^{*}\right) \wedge\left(x^{*} \vee y\right)\right) \vee z^{*}\right) \leq \\
& \leq\left(x \wedge\left((y \wedge z) \vee\left(y^{*} \wedge z^{*}\right)\right)\right) \vee\left(x^{*} \wedge\left(\left(y \wedge z^{*}\right) \vee\left(y^{*} \wedge z\right)\right)\right)
\end{aligned}
$$

then every commutative ${ }^{*}$-lattice extension of $\oplus$ is associative.

Proof If $l(x, y, z)$ and $r(x, y, z)$ denote the term on the left and right side of (1), respectively, then because of $+\in\left[+_{1},+_{2}\right]$

$$
\begin{aligned}
(x+y)+z & \leq(x+y)+{ }_{2} z=((x+y) \vee z) \wedge\left((x+y)^{*} \vee z^{*}\right) \leq \\
& \leq\left(\left(x+{ }_{2} y\right) \vee z\right) \wedge\left(\left(x+{ }_{1} y\right)^{*} \vee z^{*}\right)=l(x, y, z) \leq r(x, y, z)= \\
& =\left(x \wedge\left(y+{ }_{2} z\right)^{*}\right) \vee\left(x^{*} \wedge\left(y+{ }_{1} z\right)\right) \leq \\
& \leq\left(x \wedge(y+z)^{*}\right) \vee\left(x^{*} \wedge(y+z)\right)=x+{ }_{1}(y+z) \leq x+(y+z)
\end{aligned}
$$

From this we infer

$$
\begin{gathered}
(x+y)+z \leq x+(y+z)=(y+z)+x=(z+y)+x \\
\leq z+(y+x)=z+(x+y)=(x+y)+z
\end{gathered}
$$

and hence $(x+y)+z=x+(y+z)$.

Because (1) can also be written in terms of an equation, the ${ }^{*}$-lattices which fulfil (1) form a variety.

Theorem 4.2 In the variety of*-lattices satisfying (1) every binary term satisfying $\left(\beta_{0}\right)$ is associative and coincides with $+_{1}$ and $+_{2}$.

Proof Substituting $z=0$ in (1) implies $+_{2} \leq+_{1}$ which together with $+_{1} \leq+_{2}$ implies $+_{1}=+_{2}$. The rest follows from Corollary 2.4.

Theorem 4.3 If for $a{ }^{*}$-lattice $\mathcal{L}$ the extension $+_{1}$ or $+_{2}$ is associative then $\mathcal{L}$ satisfies (1) and hence $+_{1}=+_{2}$ is the only associative *-lattice term extension. 
Proof If $+_{1}$ is associative then

$$
x+{ }_{1} y=x+{ }_{1}\left(y^{*}+{ }_{1} 1\right)=\left(x+{ }_{1} y^{*}\right)+{ }_{1} 1=\left(x+{ }_{1} y^{*}\right)^{*}=x+{ }_{2} y .
$$

By symmetry, associativity of $+_{2}$ also implies $+_{1}=+_{2}$. Now (with the notation used in the proof of Lemma 4.1)

$$
\begin{aligned}
l(x, y, z) & =\left(\left(x+{ }_{2} y\right) \vee z\right) \wedge\left(\left(x+{ }_{1} y\right)^{*} \vee z^{*}\right)= \\
& =\left(\left(x+{ }_{1} y\right) \vee z\right) \wedge\left(\left(x+{ }_{1} y\right)^{*} \vee z^{*}\right)=\left(x+{ }_{1} y\right)+{ }_{2} z=\left(x+{ }_{1} y\right)+{ }_{1} z= \\
& =x+{ }_{1}\left(y+{ }_{1} z\right)=\left(x \wedge\left(y+{ }_{1} z\right)^{*}\right) \vee\left(x^{*} \wedge\left(y+{ }_{1} z\right)\right)= \\
& =\left(x \wedge\left(y+{ }_{2} z\right)^{*}\right) \vee\left(x^{*} \wedge\left(y+{ }_{1} z\right)\right)=r(x, y, z)
\end{aligned}
$$

Theorem 4.4 In $a^{*}$-lattice $\left(L, \vee, \wedge,{ }^{*}, 0,1\right)$ with $+_{1}=+_{2}$ this operation is associative if and only if for all $x, y, z \in L$

$$
(x \vee y) \wedge\left(x^{*} \vee y^{*}\right) \wedge z^{*} \leq x \vee\left(y \wedge z^{*}\right) \vee\left(y^{*} \wedge z\right)
$$

Proof Because of the commutativity of $+_{1},+_{1}$ is associative if and only if $\left(x+{ }_{1} y\right)+{ }_{1} z \leq x+{ }_{1}\left(y+{ }_{1} z\right)$ for all $x, y, z \in L$. Let $a, b, c \in L$. Then $\left(a+{ }_{1} b\right)+{ }_{1} c \leq a+{ }_{1}\left(b+{ }_{1} c\right)$ is equivalent to $\left(a+{ }_{1} b\right)+{ }_{1} c \leq a++_{2}\left(b+{ }_{1} c\right)$ and further, this is equivalent to

$$
\left(\left(a+{ }_{1} b\right) \wedge c^{*}\right) \vee\left(\left(a+{ }_{1} b\right)^{*} \wedge c\right) \leq\left(a \vee\left(b+{ }_{1} c\right)\right) \wedge\left(a^{*} \vee\left(b+{ }_{1} c\right)^{*}\right)
$$

Therefore

(i) $\left(a+{ }_{1} b\right) \wedge c^{*} \leq a \vee\left(b+{ }_{1} c\right)$

(ii) $\left(a+{ }_{1} b\right) \wedge c^{*} \leq a^{*} \vee\left(b+{ }_{1} c\right)^{*}$

(iii) $\left(a+{ }_{1} b\right)^{*} \wedge c \leq a \vee\left(b+{ }_{1} c\right)$

(iv) $\left(a+{ }_{1} b\right)^{*} \wedge c \leq a^{*} \vee\left(b+{ }_{1} c\right)^{*}$

hold and, conversely, (i) - (iv) together imply (3). Moreover, (i) - (iv) are equivalent.

(i) $\Rightarrow($ ii):

$\left(a+{ }_{1} b\right) \wedge c^{*}=\left(a^{*}+{ }_{1} b^{*}\right) \wedge c^{*} \leq a^{*} \vee\left(b^{*}+{ }_{1} c\right)=a^{*} \vee\left(b+{ }_{2} c\right)^{*}=a^{*} \vee\left(b+{ }_{1} c\right)^{*}$ In a similar way, using

$$
x+{ }_{i} y=x^{*}+_{i} y^{*} \text { and }\left(x+{ }_{i} y\right)^{*}=x^{*}+_{3-i} y=x+{ }_{3-i} y^{*} \text { for } i=1,2,
$$

(ii) $\Rightarrow$ (iii) $\Rightarrow$ (iv) $\Rightarrow$ (i) can be proved. Therefore (i) is equivalent to (3) and hence associativity of $+_{1}$ is equivalent to the fact that (i) holds for all $a, b, c \in L$. Because of $+_{1}=+_{2}$ (i) is nothing but inequality (2) (with $x, y, z$ replaced by $a, b, c$, respectively).

We conclude this section by mentioning some examples of *-lattices that fulfil (2): 
As shown in [6], in a ${ }^{*}$-lattice $\mathcal{L}+_{1}=+_{2}$ is equivalent to $x \wedge x^{*} \leq y \vee y^{*}$ for all $x, y \in L$. This means that the orthogonal kernel $\mathrm{OK}(\mathcal{L}):=\{x \in L \mid x \perp$ $x\}=\left\{x \in L \mid x \leq x^{*}\right\}$ is orthogonal or, equivalently, according to [6], that $\mathrm{OK}(\mathcal{L})$ is a sublattice of $\mathcal{L}$.

Example 4.5 Any distributive ${ }^{*}$-lattice $\left(L, \vee, \wedge,{ }^{*}, 0,1\right)$ with $x \wedge x^{*} \leq y \vee y^{*}$ for all $x, y \in L$ fulfils (2) since

$$
\begin{aligned}
& (x \vee y) \wedge\left(x^{*} \vee y^{*}\right) \wedge z^{*}= \\
& =\left(x \wedge x^{*} \wedge z^{*}\right) \vee\left(x \wedge y^{*} \wedge z^{*}\right) \vee\left(y \wedge x^{*} \wedge z^{*}\right) \vee\left(y \wedge y^{*} \wedge z^{*}\right) \leq \\
& \leq\left(x \vee y \vee y^{*}\right) \wedge(x \vee y \vee z) \wedge\left(x \vee z^{*} \vee y^{*}\right) \wedge\left(x \vee z^{*} \vee z\right)= \\
& =x \vee\left(y \wedge z^{*}\right) \vee\left(y^{*} \wedge z\right) .
\end{aligned}
$$

Example 4.6 (cf. [3]) Consider a *-lattice that arises by the disjoint union of a lattice $\mathcal{L}$ with smallest element and its dual $\mathcal{L}_{d}$. Every element of $\mathcal{L}_{d}$ is assumed to be over every element of $\mathcal{L}$. Let the unary operation * be defined by assigning to each element of $\mathcal{L}$ the corresponding element of $\mathcal{L}_{d}$. If $\mathcal{L}$ has a greatest element then this element may be identified with the smallest element of $\mathcal{L}_{d}$. As shown in[3], for the *-lattices arising this way $+_{1}=+_{2}$ and $+_{1}=+_{2}$ is associative.

\section{References}

[1] D. Dorninger, H. Länger and M. Maczyński, The logic induced by a system of homomorphisms and its various algebraic characterizations, Demonstratio Math., 30 (1997), 215-232.

[2] D. Dorninger, H. Länger and M. Maczyński, On ring-like structures occurring in axiomatic quantum mechanics, Österr. Akad. Wiss. Sitzungsber. II, 206 (1997), 279-289.

[3] D. Dorninger, H. Länger and M. Maczyński, On ring-like structures induced by Mackey's probability function, Rep. Math. Phys., 43 (1999), 499-515.

[4] D. Dorninger, H. Länger and M. Maczyński, Lattice properties of ring-like quantum logics, Intern. J. Theor. Phys., 39 (2000), 1015-1026.

[5] D. Dorninger, H. Länger and M. Maczyński, Concepts of measures on ringlike quantum logics, Rep. Math. Phys., 47 (2001), 167-176.

[6] D. Dorninger, H. Länger and M. Maczyński, Ring-like structures with unique symmetric difference related to quantum logic, Discussiones Math., 21 (2001), 239-253. 
[7] D. Dorninger and D. Schweigert, Zur Darstellung von Polynomen auf De Morgan Algebren, Czechoslovak Math. J., 30 (1980), 65-70.

[8] H. Lausch and W. Nöbauer, Algebra of polynomials, North-Holland, Amsterdam, and Amer. Elsevier, New York, 1973.

[9] M. Navara, On generating finite orthomodular sublattices, Tatra Mt. Math. Publ., 10 (1997), 109-117.

Received: February 15, 2007 